Ann. Zootech., I963, 12 (I), 39-52

\title{
COMPOSITION CHIMIQUE DE' L'HERBE ET TÉTANIE D'HERBAGE
}

\author{
P. LARVOR et L. GUÉGUEN \\ avec la collaboration technique de Nicole Berthelot et de Michelle Frasnier-Langluis \\ Laboratoire de Nutrition minérale, Ëcole nationale vétérinaire, Alfort (Seine); \\ Laboratoire des Métabolismes, Centre national de Recherches zootechniques, \\ Jouy-en-Josas (Seine-et-Oise)
}

\section{SOMMAIRE}

Deux séries de recherches ont été effectuées sur les relations entre la composition chimique de l'herbe et les symptômes de Tétanie d'herbage :

$\mathrm{I}^{\mathrm{o})}$ On a comparé, au moyen de la fonction discriminante de R. A. Fisher, la composition de deux groupes d'herbes prélevées au printemps r960. Le premier groupe se composait de 35 échantillons provenant de prairies normales, et le second de 45 échantillons provenant de prairies tétanigènes. Les analyses ont été effectuées pour la matière sèche, le calcium, le phosphore, le magnésium, le potassium, le sodium, la cellulose brute et l'azote non élaboré.

Les herbes tétanigènes se sont distinguées par une teneur plus faible en magnésium et en cellulose brute, et par une teneur plus élevée en azote non élaboré.

$\left.2^{0}\right)$ On a établi l'équation de régression multiple reliant le magnésium plasmatique des vaches laitières à la composition de l'herbe qu'elles consommaient au printemps $196 \mathrm{r}$ (29 pâtures). Les analyses ont été effectuées pour la matière sèche, le calcium, le phosphore, le magnésium, le potassium, le sodium, la cellulose brute, les matières azotées totales et l'azote soluble.

Les herbes qui contenaient le plus d'azote soluble correspondaient aux taux du magnésium plasmatique les plus faibles (coefficient de corrélation $r=-0,543 ; \mathrm{P}<0,0 \mathrm{I}$ ).

Les résultats de ces deux séries de prélèvements ont été comparés et discutés.

Parmi les nombreux aspects que présente le problème de l'étiologie de la Tétanie d'herbage, la question de la composition chimique de 1'herbe tétanigène a déjà suscité de nombreux travaux contradictoires.

Pour un premier groupe de chercheurs, la composition minérale de l'herbe suffit à caractériser son pouvoir tétanigène. BRouwER et Van de VLIERT (I95I) et BrouWER (I95I-I952) trouvent une relation étroite entre l'incidence de la Tétanie et la différence " alcalinité alcaline " — " alcalinité alcalino-terreuse " dans la matière 
sèche de 1'herbe, soit $(\mathrm{K}+\mathrm{Na}-\mathrm{Cl}-\mathrm{S})-(\mathrm{Ca}+\mathrm{Mg}-\mathrm{P})$ calculé en milliéquivalents. VERDEYEN (I953) observe un parallélisme entre la composition de l'herbe en " potasse non antagonisée " et les années à Tétanie, la "potasse non antagonisée " étant la différence $\mathrm{K}_{2} \mathrm{O}-(\mathrm{CaO}+\mathrm{MgO})$. 't HART et $\mathrm{KEMP}$ (I956) calculent différentes formules pour caractériser l'influence tétanigène d'une herbe; nous citerons l'une des plus intéressantes, parce qu'obtenue selon des méthodes objectives (régression multiple) :

$\log (\mathrm{KZ}+\mathrm{I})=3, \mathrm{I} 35 \log \mathrm{K}_{2} \mathrm{O}-\mathrm{O,II} 6 \log \mathrm{Na}_{2} \mathrm{O}-\mathrm{I}, \mathrm{I} 2 \mathrm{I} \log \mathrm{CaO}-2,028$ ( $\mathrm{KZ}=$ fréquence $\mathrm{p}$. Ioo de la tétanie $; \mathrm{K}_{2} \mathrm{O}, \mathrm{Na}_{2} \mathrm{O}$ et $\mathrm{CaO}$ sont exprimés en $\mathrm{p}$. Ioo de la matière sèche) ; ils ont observé avec cette formule un coefficient de corrélation intéressant $(r=0,794)$ entre la fréquence prévue de la Tétanie et la composition de l'herbe. Cependant les mêmes auteurs (KEMP et 't HART, 1957) préfèrent le rapport $\mathrm{K} / \mathrm{Ca}+\mathrm{Mg}$ calculé en milliéquivalents; avec les échantillons qu'ils ont étudiés, on commence à observer quelques cas de Tétanie, lorsque ce rapport est supérieur à $\mathrm{I}, 8$, mais c'est à partir de 2,2 que l'incidence de la Tétanie devient forte ( 5 p. Ioo), et lorsque ce rapport devient supérieur à 3 , ils observent plus de 17 p. Ioo de cas.

D'autres chercheurs, cependant, ont essayé de reproduire ces résultats, avec des fortunes diverses. NAUmanN et BARTH (I959) proposent une formule complétée:

$\frac{\mathrm{K}}{\mathrm{Ca}+\overline{\mathrm{Mg}}}+\frac{\mathrm{IOO}}{\overline{\mathrm{Mn}}}+\frac{\mathrm{IO}}{\mathrm{Cu}}$ (Mn et $\mathrm{Cu}$ étant exprimés en $\mathrm{mg} / \mathrm{kg}$ de matière sèche) et estiment que la valeur de ce rapport ne devrait pas dépasser 3,5 sans danger de Tétanie.

Pat contre Werner (I959) en Allemagne, Rook et WoOD (I960) et Rook et BALCH (1962) en Angleterre n'observent que des relations très lointaines entre la composition minérale de l'herbe et les manifestations de Tétanie, ou le magnésium sérique des animaux. ODELIEN (Ig60) en Norvège et PETERs (I960) en Allemagne ont adopté ces conclusions.

Cependant, en Hollande même, 1'importance du rapport $\mathrm{K} / \mathrm{Ca} \div \mathrm{Mg}$ a récemment été mise en doute (SEEKIES, I958, BAKKER, I960, HENDRIKS, I962) et KEMP (I960) a également revisé ses positions et fait désormais intervenir trois facteurs principaux : le magnésium, le potassium et les protéines de l'herbe.

Dans le domaine des constituants organiques de l'herbe, INGris (Ig60) a fait remarquer que l'évolution de la magnésiémie chez des moutons au pâturage, suivait une courbe analogue à celle de la teneur en cellulose de $1^{\prime}$ herbe, et inverse de celle de la teneur en matière azotées totales; néanmoins NAUMANN et BARTH (I959) n'ont rien observé de ce côté.

Enfin BurT et Thomas (I96I) ont suggéré la possibilité du rôle des citrates de l'herbe dans l'étiologie de la Tétanie.

\section{MATÉRIEI, ET MÉTHODES}

Les observations ont été effectuées pendant deux années consécutives avec un matériel et des méthodes sensiblement différents. 
$\mathrm{I}^{\mathrm{o})}$ Prélèvements.

Au printemps I960, nous avons demandé à un certain nombre de vétérinaires de nous envoyer des échantillons d'herbe récoltés dans des prairies ayant provoqué ou non de la 'Tétanie d'herbage ; I 20 échantillons furent analysés dont 35 provenant de prairies normales (Ile-de-France, Normandie et Nord) et 85 provenant de prairies à Tétanie d'herbage. Dès leur réception, ces 85 échantillons furent classés en "sûrement tétanigènes " (45) et en "douteux " (40). Ne furent considérés comme "sûrement tétanigènes " que les échantillons provenant de régions à forte fréquence de Tétanie d'herbage, ayant provoqué des symptômes absolument typiques, et récoltés le jour même de la crise.

Au printemps 196r,! es échantillons d'herbe furent prélevés dans une région beaucoup plus res* treinte des Flandres, et chaque échantillon d'herbe correspondait à un ou plusieurs échantillons de plasma prélevés simultanément. Les conditions de prélèvement et l'interprétation des analyses de ces échantillons de plasma ont déjà fait l'objet d'une publication (LARvor, I962).

\section{$\left.2^{\circ}\right)$ Dosages.}

La matière sèche fut déterminée par pesée après séchage à $100^{\circ} \mathrm{C}$ en 1960 , et à $70^{\circ} \mathrm{C}$ en $196 \mathrm{r}$. Les éléments minéraux furent dosés après minéralisation au four à $55^{\circ} \mathrm{C}$ : le calcium par complexométrie en 1960 et par photométrie de flamme (appareil Lippendorf) en I96r ; le phosphore par la méthode de Misson au phosphovanadomolybdate d'ammonium, le magnésium par le jaune de titane en 1960 et par photométrie de flamme en I96I (appareil Eppendorf, technique de (iUÉGUEN et Rombauts, 196r), le sodium et le potassium par photométrie de flamme (appareil Jobin et Yron en I960, appareil Eppendorf en 1961).

La cellulose brute (WEENDE) fut dosée par la méthode rapide préconisée par l'A. F. Z. ; cette méthode remplace les lavages jusqu'à cessation de réaction acide ou alcaline par une neutralisation en présence de bleu de bromothymol, ce qui réduit considérablement le nombre de centrifugations ( $\left.{ }^{(}\right)$.

Pour la teneur en matières azotées de l'herbe, on a essayé en rg6o d'évaluer quantitativement les produits faiblement élaborés, au moyen de la méthode arbitraire suivante, dont les résultats se sont montrés reproductibles:

Le produit étant très finement broyé (consistance de farine), on en pèse $0,5 \mathrm{~g}$ que l'on place dans un tube à essai avec Io $\mathrm{ml}$ d'eau. On agite $15 \mathrm{mn}$ puis on défèque avec $2,5 \mathrm{ml}$ d'acide trichloracétique à $20 \mathrm{p}$. Ioo. On filtre sur papier en lavant 2 fois le filtre avec Io $\mathrm{ml}$ d'eau en tout. Le filtrat est placé dans l'appareil à entraînement par la vapeur d'eau (appareil de PREGL), on ajoute Io $\mathrm{ml}$ de carbonate de soude en solution saturée. L'ammoniaque produit est distillé à l'aide d'un courant de vapeur d'eau à $100^{\circ} \mathrm{C}$, le produit de distillation est condensé et recueilli dans un erlenmeyer contenant $20 \mathrm{ml}$ de $\mathrm{SO}_{4} \mathrm{H}_{2} \mathrm{o}, 02 \mathrm{~N}$. L'ammoniaque est ensuite dosé par colorimétrie en présence de réactif de Nessler, à 520 mu. Les résultats sont exprimés en $\mathrm{N}$ p. I 000 de la matière sèche et seront désignés sous le nom d'azote non élaboré (A. N. E.).

En I96r, ce dosage a été remplacé par celui des matières azotées totales (méthode Kjeldahl) ( ${ }^{1}$ ) et par le dosage de l'azote soluble dans l'éthanol à 80 p. 100 à la température ambiante ( ${ }^{(2)}$.

\section{$\left.3^{\circ}\right)$ Statistiques.}

Pour les résultats de t96o, il fallait comparer deux groupes qualitativement différents, définis chacun par 8 variables (matière sèche, calcium, phosphore, magnésium, sodium, potassium, cellulose brute, azote non élaboré), le problème étant de trouver la fonction linéaire de ces 8 variables qui différencie au mieux les 2 groupes de données (herbes normales et herbes tétanigènes). U'ne telle fonction peut être écrite sous la forme :

$$
\text { 'T }=f(\text { M. S., Ca, P, Mg, Na, K, C. B., A. N. I.). }
$$

Cette fonction discriminante (R. A. FISHER) est une nouvelle variable caractéristique de chaque échantillon d'herbe, ses coefficients sont calculés de telle façon que la valeur du $t$ de Student-Fisher entre les moyennes $\overline{T^{\prime}}$ et $\overline{T^{\prime \prime}}$ de cette fonction pour les deux populations d'herbe soit maximum.

Les calculs ont été effectués d'après DELTHEIL et HURON (1959) sur calculateur électronique Bull, Gamma ET.

Pour les résultats de I96I, le problème était très différent. Il fallait établir une relation entre la composition de l'herbe consommée et le magnésium plasmatique des animaux, qui peut être

(1) Dosages de cellulose et d'azote total 1961 effectués par le Laboratoire d'Essai et d'Analyse des Aliments, dépendant de la Station centrale de Nutrition.

( ${ }^{2}$ Dosages d'azote soluble effectués par Madeleine Forignon, Laboratoire des Métabolismes, C. N. R. Z. 
considéré comme une variable continue. Dans ces conditions, s'impose le calcul de l'équation de régression multiple entre le magnésium plasmatique d'une part, et les différents composants des herbes d'autre part (M. S., Ca, P, Mg, Na, K, C. B., P'rotéines brutes, Azote soluble). Ce travail a été effectué sur calculateur électronique I. B. M. 7090.

\section{RÉSULTATS}

\section{$\left.\mathbf{I}^{\circ}\right)$ Données recueillies au printemps I960}

Le tableau I rassemble les résultats des analyses. Les herbes classées comme "douteuses ", n'ayant pas servi au calcul de la fonction discriminante, ne figurent pas dans ce tableau.

Pour rendre la répartition des variables approximativement conforme à une courbe de Gauss, on a adopté les transformations suivantes:

Matière sèche $p$. Ioo

Calcium p. I ooo de la M. S.

Phosphore p. I ooo de la M. S

Magnésium p. I ooo de la M. S.

Sodium p. I ooo de la M. S.

Potassium p. I ooo de la M. S
$=\mathrm{M} . \mathrm{s}$. racine carrée

$=\mathrm{Ca}$ logarithme décimal

$=\mathrm{P}$

$=\mathrm{Mg}$

$=\mathrm{Na}$

$=\mathrm{K}$ racine cubique

logarithme décimal

racine cubique

sans transformation

Azote non élaboré p. Ioo de la M. S. $=$ A.N.E. logarithme décimal

Les calculs indiqués ci-dessus nous ont fourni l'équation suivante, dans laquelle les différents paramètres ont été rangés par ordre d'importance décroissante :

$$
\begin{aligned}
\mathrm{T}=-6,82 \log \text { A.N.E. }+ \text { o,4I I C.B. } & +\mathrm{I} 5,67 \log \mathrm{Mg}-8,60 \sqrt[3]{\mathrm{P}}-4,08 \sqrt[3]{\mathrm{Na}} \\
& +0,769 \sqrt[2]{\mathrm{M} . \mathrm{S} .}+0,052 \mathrm{~K}-2,48 \log \mathrm{Ca} .
\end{aligned}
$$

Cette formule peut être considérablement simplifiée :

$\mathrm{I}^{\circ}$ ) en supprimant les différents facteurs qui n'apportent pas à l'efficacité de la formule une amélioration statistiquement significative. Cette suppression a été réalisée selon une méthode régressive, c'est-à-dire en recalculant chaque fois la fonction après élimination successive des différentes variables, et en testant chaque fois, par une analyse de variance, la signification de la variance contrôlée par la variable éliminée ;

$2^{\circ}$ ) en remplaçant $\log$ A. N. E. par $\log$ Ioo A. N. E., ce qui évite d'avoir à manipuler des logarithmes négatifs;

$3^{\circ}$ ) en prenant arbitrairement 1'un des coefficients de pondération comme égal à l'unité, ce qui impose une multiplication des autres coefficients par un même facteur.

On obtient alors l'équation stivante :

$$
\mathrm{T}=38, \mathrm{x} \log \mathrm{Mg}+\text { C. B. }-\mathrm{I} 6,6 \log \text { I00 A. N. E., }
$$

clans laquelle $\mathrm{Mg}$ est exprimé en p. I ooo de 1a matière sèche, C. B. est la cellulose brute en p. Ioo de la matière sèche et A. N. F.. l'azote non élaboré en p. Iooo de la matière sèche. 
La figure I permet de comparer l'histogramme de $\mathrm{K} / \mathrm{Ca}+\mathrm{Mg}$ et 1'histogramme des valeurs de la fonction discriminante ( $T$ ) pour les herbes tétanigènes, normales ou douteuses. Elle permet de constater que, dans le cadre de notre échantillonnage, $\mathrm{K} / \mathrm{Ca}+\mathrm{Mg}$ n'offre aucune valeur de discrimination, tandis que la fonction discrimi-
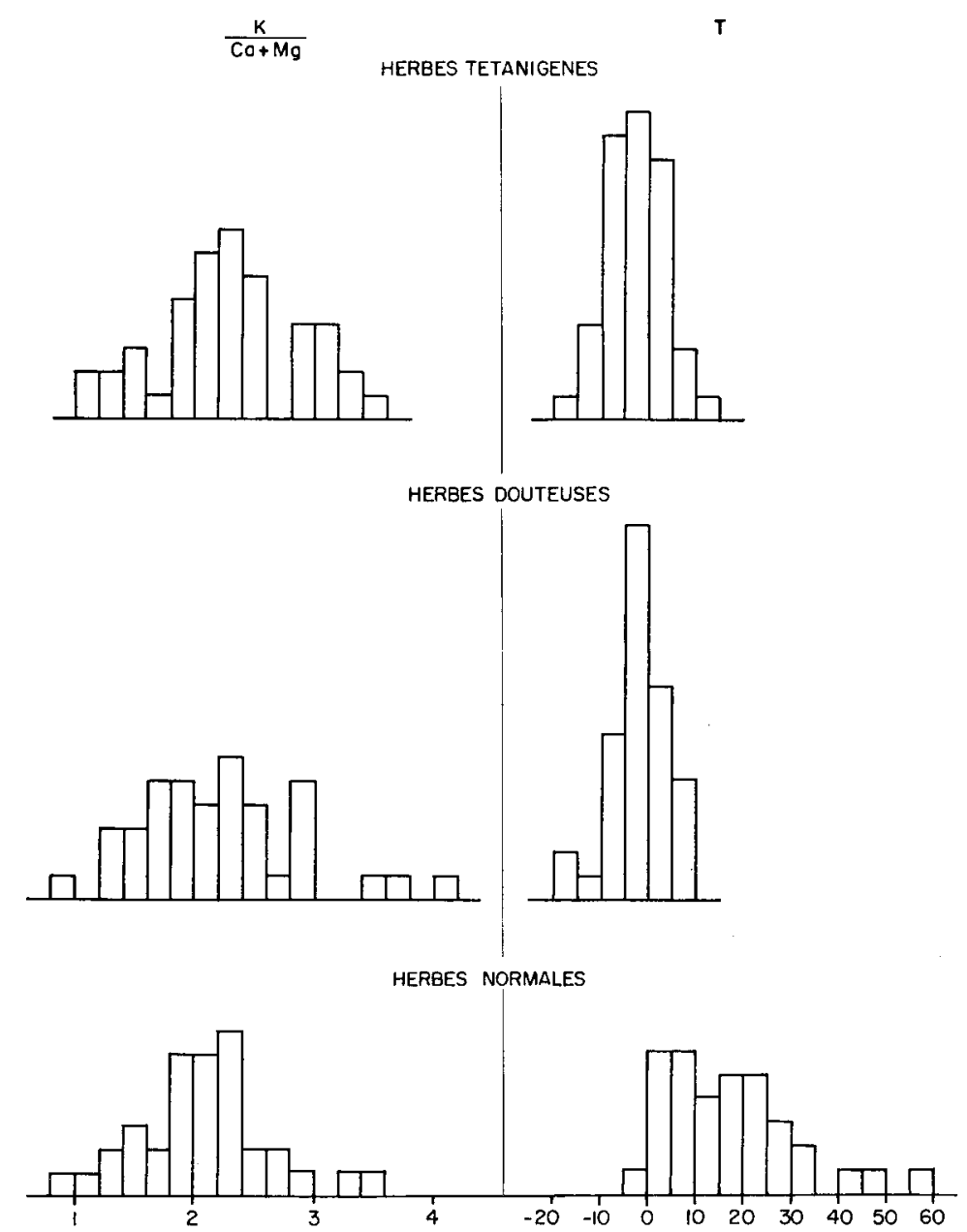

FIG. I. - Histogramme des valeurs de $\mathrm{K} / \mathrm{Ca}+\mathrm{Mg}$ el de $\mathrm{T}$ pour différentes catégories d'herbes

nante permet dans une certaine mesure de différencier herbes normales et herbes tétanigènes. La correspondance étroite entre 1'histogramme des valeurs de cette fonction pour les herbes tétanigènes et les herbes douteuses semble indiquer que 1'élimination des herbes "douteuses » a été d'une sévérité excessive.

$\left.2^{\circ}\right)$ Données recueillies au printemps Ig6I

Le tableau 2 rassemble les résultats des analyses d'herbe, avec le taux de magnésium plasmatique correspondant. Celui-ci a été calculé de la façon suivante : 


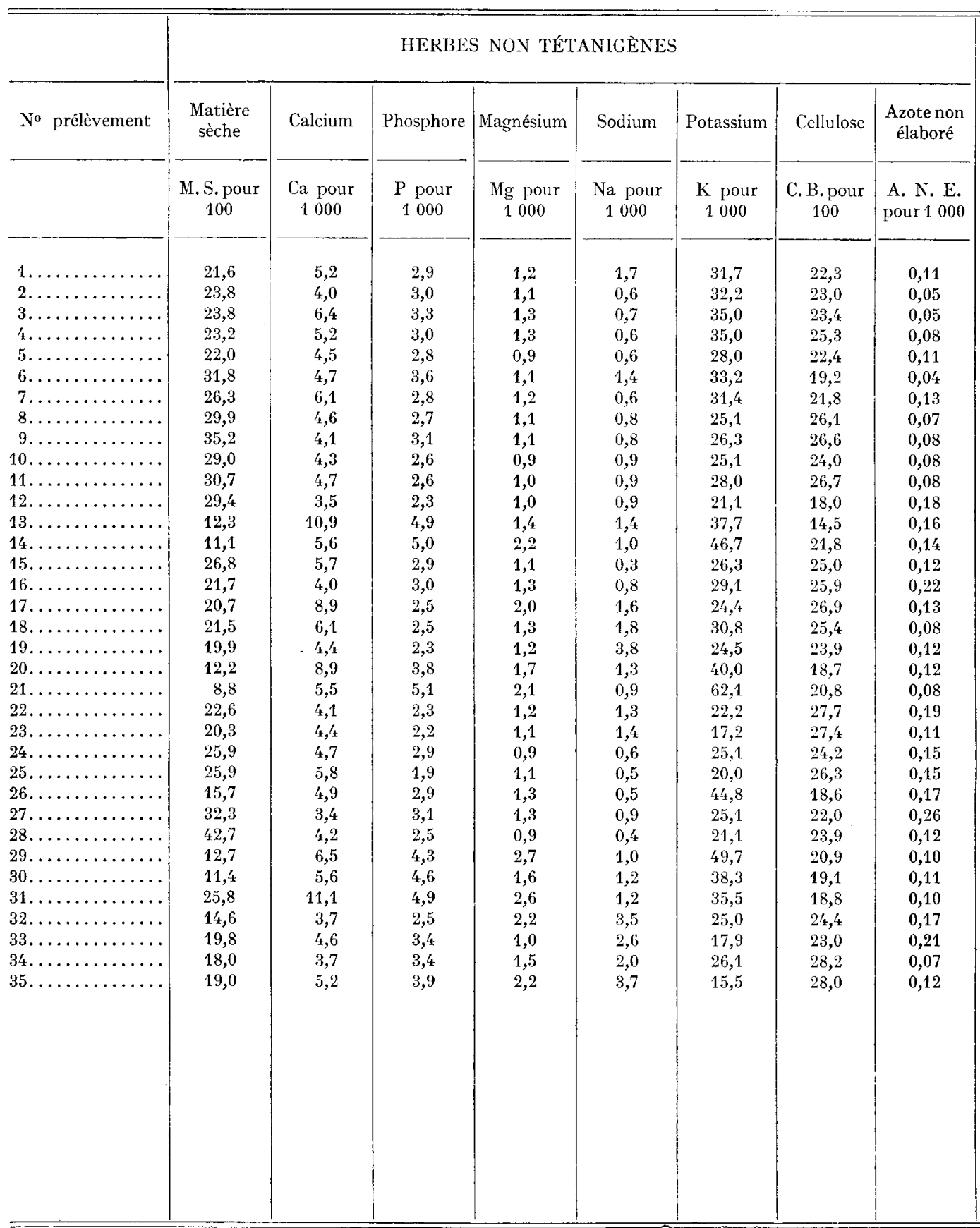


AU I

tétanigènes recueillies au printemps 1960

la matière sèche)

\begin{tabular}{|c|c|c|c|c|c|c|c|c|}
\hline \multirow[b]{2}{*}{ No prélèvement } & \multicolumn{8}{|c|}{ HERBES TÉTANIGENES } \\
\hline & $\begin{array}{c}\text { Matière } \\
\text { sèche }\end{array}$ & Calcium & Phosphore & Magnésium & Sodium & Potassium & Cellulose & $\begin{array}{c}\text { Azote non } \\
\text { élaboré }\end{array}$ \\
\hline & $\begin{array}{c}\text { M.S. pour } \\
100\end{array}$ & $\begin{array}{c}\mathrm{Ca} \text { pour } \\
1000\end{array}$ & $\begin{array}{l}P_{\text {pour }} \\
1000\end{array}$ & $\begin{array}{c}\mathrm{Mg} \text { pour } \\
\mathbf{1} 000\end{array}$ & $\begin{array}{c}\mathrm{Na} \text { pour } \\
1000\end{array}$ & $\begin{array}{c}K_{1} \text { pour } \\
000\end{array}$ & $\begin{array}{c}\text { C. B. pour } \\
100\end{array}$ & $\begin{array}{l}\text { A. N. E. } \\
\text { pour } 1000\end{array}$ \\
\hline$\ldots \ldots \ldots$ & 17,8 & 6,4 & 4,4 & 2,0 & 0,6 & 36,6 & 14.7 & 1.03 \\
\hline $37 \ldots \ldots \ldots \ldots \ldots$ & 13,7 & 7,7 & 4,5 & 0,8 & 1,4 & 38,2 & 19,0 & 0,18 \\
\hline $38 \ldots \ldots \ldots \ldots \ldots$ & 13,0 & 8,2 & 4,8 & 1,9 & 2,6 & 29,4 & 15,0 & 0,06 \\
\hline $39 \ldots \ldots \ldots \ldots \ldots$ & 15,4 & 5,4 & 4,5 & 0,9 & 0,6 & 34,3 & 19,3 & 0,24 \\
\hline $40 \ldots \ldots \ldots \ldots \ldots$ & 14,3 & 4,9 & 3,4 & 1.7 & 0,8 & 37,8 & 20,5 & 1,21 \\
\hline $41 \ldots \ldots \ldots \ldots$ & 17,3 & 5,1 & 5,0 & 1,4 & 0,6 & 44,1 & 22,8 & 1,18 \\
\hline $42 \ldots \ldots \ldots \ldots$ & 15,4 & 6,4 & 7,1 & 1,7 & 2,0 & 36,1 & 18,6 & 1,22 \\
\hline $43 \ldots \ldots \ldots \ldots \ldots$ & 13,7 & 7,9 & 4,8 & 1,0 & 3,3 & 27,0 & 21,0 & 0,30 \\
\hline $44 \ldots \ldots \ldots \ldots \ldots$ & 19,6 & 5,5 & 3,3 & 1,2 & 1,3 & 33,9 & 16,4 & 0,30 \\
\hline $45 \ldots \ldots \ldots \ldots \ldots$ & 18,6 & 5,9 & 3,7 & 1,0 & 3,0 & 32,8 & 19,5 & 0,70 \\
\hline $46 \ldots \ldots \ldots \ldots \ldots$ & 20,2 & 6,3 & 3,1 & 1,4 & 0,9 & 32,8 & 16,4 & 0,12 \\
\hline $47 \ldots \ldots \ldots \ldots \ldots$ & 17,0 & 7,7 & 3,5 & 1,5 & 3,1 & 23,4 & 18,0 & 0,34 \\
\hline $48 \ldots \ldots \ldots \ldots$ & 16,8 & 6,0 & 4,8 & 1,1 & 1,1 & 34,4 & 17,0 & 0,39 \\
\hline $49 \ldots \ldots \ldots \ldots$ & 18,9 & 5,1 & 3,1 & 1,2 & 2,2 & 32,4 & 16,8 & 0,21 \\
\hline $50 \ldots \ldots \ldots \ldots$ & 18,4 & 6,1 & 5,0 & 1,7 & 1,7 & 35,0 & 18,1 & 0,27 \\
\hline $51 \ldots \ldots \ldots \ldots \ldots$ & 17,2 & 11,3 & 2,8 & 0,9 & 1,5 & 34,4 & 14,6 & 0,31 \\
\hline $52 \ldots \ldots \ldots \ldots \ldots$ & 16,6 & 4,6 & 4,6 & 1,2 & 2,0 & 32,2 & 22,0 & 0,28 \\
\hline $53 \ldots \ldots \ldots \ldots \ldots$ & 12,2 & 6,9 & 3,7 & 1,0 & 1,2 & 40,6 & 21,0 & 0,40 \\
\hline $54 \ldots \ldots \ldots \ldots \ldots$ & 16,4 & 5,2 & 4,2 & 1,0 & 1,5 & 34,1 & 18,0 & 0,22 \\
\hline $55 \ldots \ldots \ldots \ldots \ldots$ & 16,9 & 5,6 & 3,0 & 1,8 & 8,5 & 25,5 & 17,8 & 0,24 \\
\hline $56 \ldots \ldots \ldots \ldots \ldots$ & 20,5 & 4,9 & 5,6 & 1,8 & 2,9 & 40,0 & 21,8 & 0,70 \\
\hline $57 \ldots \ldots \ldots \ldots \ldots$ & 13,2 & 6,6 & 5,2 & 1,9 & 0,8 & 36,3 & 21,1 & 0,58 \\
\hline $58 \ldots \ldots \ldots \ldots \ldots$ & 18,8 & 6,6 & 3,9 & 0,9 & 1,2 & 32,1 & 21,9 & 0,18 \\
\hline $59 \ldots \ldots \ldots \ldots \ldots$ & 16,5 & 5,7 & 4,2 & 0,8 & 2,0 & 40,0 & 16,2 & 0,34 \\
\hline $60 \ldots \ldots \ldots \ldots \ldots$ & 23,5 & 6,5 & 3,5 & 1,0 & 1,5 & 24,8 & 15,8 & 0,14 \\
\hline $61 \ldots \ldots \ldots \ldots \ldots$ & 23,4 & 10,7 & 4,9 & 1,4 & 0,6 & 45,8 & 20,5 & 0,66 \\
\hline $62 \ldots \ldots \ldots \ldots \ldots$ & 20,8 & 6,1 & 4,0 & 0,9 & 3,0 & 30,9 & 21,1 & 0,21 \\
\hline $63 \ldots \ldots \ldots \ldots$ & 20,7 & 5,5 & 3,4 & 1,2 & 0,5 & 32,1 & 22,4 & 0,17 \\
\hline $64 \ldots \ldots \ldots \ldots \ldots$ & 23,0 & 6,6 & 3,2 & 1,3 & 1,3 & 29,1 & 19,2 & 0,15 \\
\hline $65 \ldots \ldots \ldots \ldots \ldots$ & 20,1 & 7,5 & 2,9 & 1,2 & 1,6 & 21,7 & 13,5 & 0,15 \\
\hline $66 \ldots \ldots \ldots \ldots \ldots$ & 21,8 & 5,1 & 2,7 & 0,8 & 1,2 & 25,3 & 17,8 & 0,08 \\
\hline $67 \ldots \ldots \ldots \ldots$ & 26,8 & 4,3 & 2,7 & 0,9 & 0,6 & 34,1 & 17,3 & 0,14 \\
\hline $68 \ldots \ldots \ldots \ldots \ldots$ & 20,7 & 4,3 & 3,0 & 0,8 & 2,5 & 35,9 & 13,1 & 0,14 \\
\hline $69 \ldots \ldots \ldots \ldots \ldots$ & 24,2 & 4,9 & 2,9 & 0,9 & 1,3 & 24,7 & 19,1 & 0,15 \\
\hline $70 \ldots \ldots \ldots \ldots$ & 22,5 & 5,7 & 3,1 & 0,9 & 1,7 & 31,2 & 16,7 & 0,22 \\
\hline $71 \ldots \ldots \ldots \ldots$ & 15,8 & 4,8 & 3,0 & 0,9 & 3,0 & 37,6 & 16,7 & 0,51 \\
\hline $72 \ldots \ldots \ldots \ldots \ldots$ & 19,3 & 3,8 & 3,3 & 0,9 & 1,9 & 32,3 & 23,4 & 0,27 \\
\hline $73 \ldots \ldots \ldots \ldots \ldots$ & 21,0 & 3,8 & 3,0 & 0,7 & 1,5 & 34,7 & 16,6 & 0,17 \\
\hline $74 \ldots \ldots \ldots \ldots \ldots$ & 18,4 & 4,7 & 3,4 & 1,2 & 1,2 & 24,3 & 25,0 & 0,28 \\
\hline $75 \ldots \ldots \ldots \ldots$ & 20,0 & 6,4 & 4,3 & 0,7 & 0,6 & 46,0 & 17,7 & 0,47 \\
\hline $76 \ldots \ldots \ldots \ldots$ & 15,4 & 5,2 & 3,2 & 1,2 & 2,9 & 32,2 & 24,0 & 0,15 \\
\hline $77 \ldots$ & 17,2 & 3,8 & 4,0 & 1,0 & 1,6 & 32,8 & 21,0 & 0,26 \\
\hline $78 \ldots \ldots \ldots$ & 19,0 & 4,5 & 3,2 & 1,1 & 1,5 & 29,4 & 24,9 & 0,13 \\
\hline $79 \ldots \ldots \ldots$ & 12,3 & 5,2 & 4,9 & 1,1 & 2,3 & 39,4 & 24,2 & 0,33 \\
\hline $80 \ldots \ldots \ldots \ldots \ldots$ & 21,7 & 7,0 & 3,7 & 0,9 & 2,3 & 30,6 & 22,5 & 0,13 \\
\hline
\end{tabular}


Une étude antérieure (LARVOR, I962) ayant montré pour ce même ensemble de données une relation nette entre le magnésium plasmatique et le stade de lactation $(r=+0,506)$, on a calculé pour chaque vache la valeur théorique de son magnésium plasmatique en supposant qu'elle soit à roo jours de lactation, ce qui élimine un important facteur de variation.

Pour chaque herbe on a ensuite calculé la moyenne des magnésium plasmatiques corrigés des différentes vaches qui la consommaient ( I à 3 prélèvements selon les cas).

Les transformations et symboles suivants ont été adoptés :

\begin{tabular}{|c|c|c|c|}
\hline Potassium & $=\mathrm{K}$ & p. I ooo de la M. S. & sans transformation \\
\hline Calcium & $=\mathrm{Ca}$ & p. I ooo de la M. S. & logarithme \\
\hline Magnésium & $=\mathrm{Mg}$ & p. I 000 de la M. S. & logarithme \\
\hline Phosphore & $=\mathrm{P}$ & p. I ooo de la M. S. & sans transformation \\
\hline Sodium & $=\mathrm{Na}$ & p. I ooo de la M. S. & racine cubique \\
\hline Matière sèche & $=$ M.S. $\mathrm{p}$ & p. Ioo de la M. S. & racine carrée \\
\hline Cellulose brute & $=\mathrm{C} \cdot \mathrm{B} \cdot \mathrm{p}$ & de la M. S. & sans transformation \\
\hline Matière azotées totales & $=\mathrm{M} \cdot \mathrm{A} \cdot \mathrm{p}$ & de la M. S. & logarithme \\
\hline Azote soluble $\times 6,25$ & $=$ N.S. $\mathrm{p}$ & de la M. S. & logarithme \\
\hline
\end{tabular}

$\mathrm{Mg}$ plasmatique moyen

à Ioo jours de lactation $=\mathrm{Y} \mathrm{mg}$ p. Ioo $\mathrm{ml}$

logarithme

Sur certains échantillons l'azote soluble n'a pu ètre dosé, aussi avons nous calculé d'abord la régression multiple entre le magnésium plasmatique et les composants de l'herbe sans tenir compte de l'azote soluble.

Dans ces conditions, on obtient la régression :

$$
\begin{array}{r}
\mathrm{Ln} \mathrm{Y}=\mathrm{I} 5,5-0,024 \mathrm{~K}-\mathrm{I}, 32 \mathrm{LnCa}-0,68 \mathrm{I} \mathrm{LnMg}+0,202 \mathrm{P}-0,427 \sqrt[3]{\mathrm{Na}} \\
-0,570 \sqrt[2]{\mathrm{M} . S .}-0, \mathrm{I} 7 \mathrm{C} \cdot \mathrm{B} .-2,59 \mathrm{Ln} \text { PRO. }
\end{array}
$$

Le coefficient de corrélation multiple est $\mathrm{R}=0,8 \mathrm{I} 4$ pour $\mathrm{I} 8$ degrés de liberté. Si l'on réduit cette équation aux variables dont le coefficient de régression partiel est significatif, et que l'on transforme les logarithmes népériens en logarithmes décimaux, on obtient :

$$
\log \mathrm{Y}=7, \mathrm{I} 79-\mathrm{I}, 97 \log \mathrm{PRO}-0,067 \mathrm{CB}
$$

Le coefficient de corrélation multiple est alors $R=0,693$ pour 24 degrés de libe:té (limite I p. Ioo $=0,565$ ).

Si on introduit dans les calculs la variable azote soluble, on obtient avec le

\begin{tabular}{|c|c|c|c|}
\hline N Soluble & N Total & cellulose brute & \\
\hline \multirow[t]{2}{*}{$-0,54361\left(^{* *}\right)$} & - $0,48969\left(^{*}\right)$ & $-0,06659$ & Mg plasmat. \\
\hline & $+0,37657$ & $+0,06943$ & $\mathrm{~N}$ soluble \\
\hline \multicolumn{2}{|c|}{$\begin{aligned} * \mathrm{P} & <0,05 \text { (limite }=0,423) \\
* * \mathrm{P} & <0,01 \text { (limite }=0,537)\end{aligned}$} & $-0,62824(* *)$ & $\mathrm{N}$ Total \\
\hline
\end{tabular}
magnésium plasmatique les corrélations simples suivantes (pour 22 échantillons seulement, puisque l'Azote soluble n'a pas été dosé dans tous les cas) : 


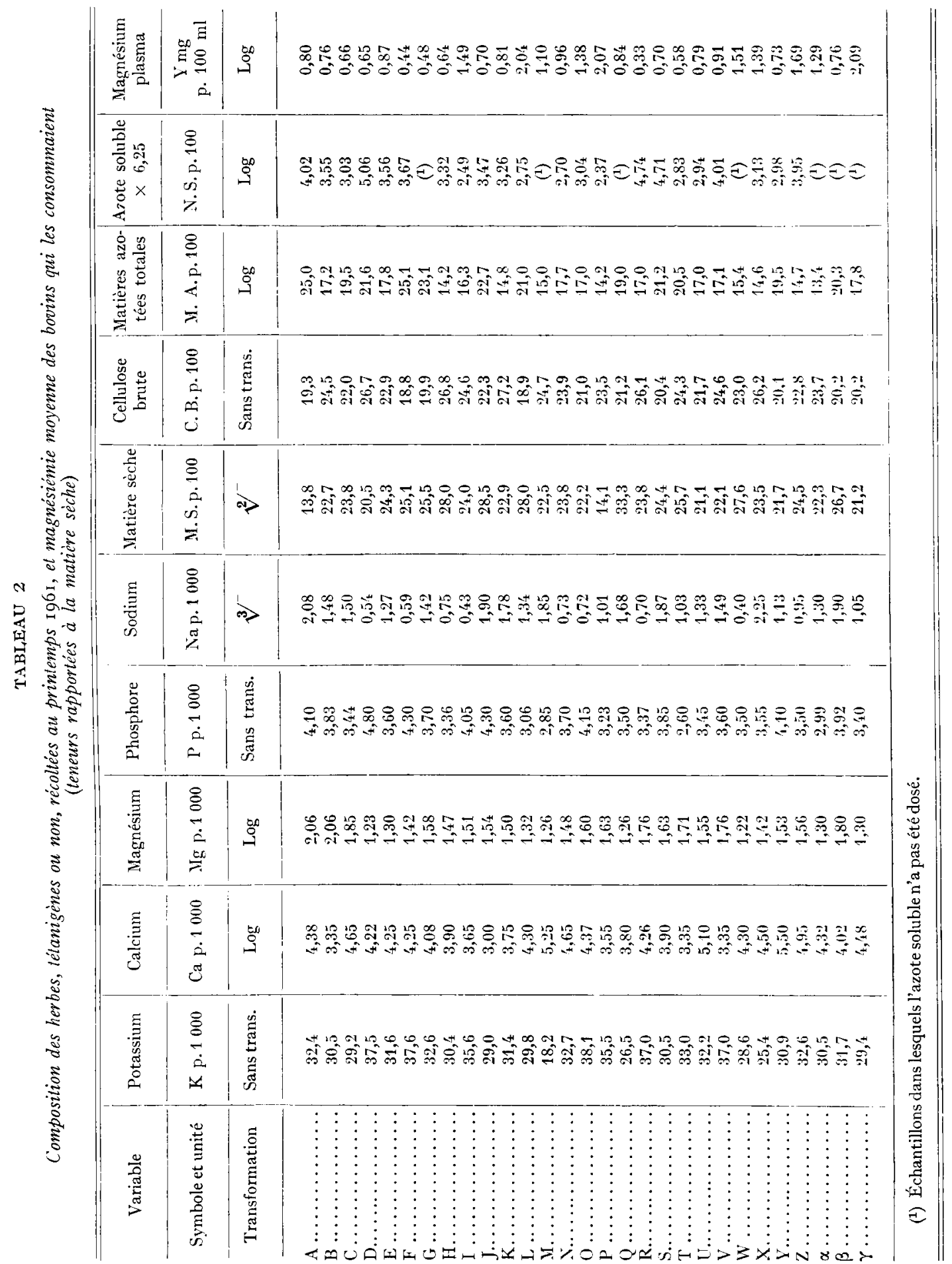


Il en découle les corrélations multiples suivantes entre le magnésium plasmatique et ces trois facteurs pris ensemble ou deux par deux :

Avec Azote total, Cellulose brute et Azote soluble $\mathrm{R}=0,689$ (limite $\mathrm{P}=0,0 \mathrm{I}: 0,678$ )

Azote total et Cellulose brute $\mathrm{R}=0,638$

Azote total et Azote soluble $\mathrm{R}=0,549$ la limite $\mathrm{P}=0,05$ est de 0,520 ;

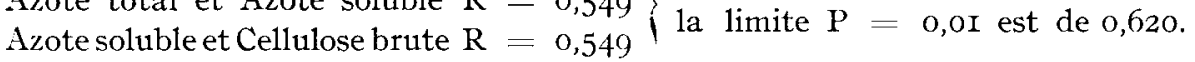

On constate donc que l'azote soluble est en corrélation hautement significative avec le magnésium plasmatique, corrélation dont la probabilité de réalisation aléatoire est à peu près aussi faible que celle de la corrélation multiple du magnésium plasmatique avec les protéines et la Cellulose brute.

Nous le considérerons donc comme le facteur principal. Il en résulte que ni les protéines, ni la Cellulose brute n'apportent de précision supplémentaire.

On peut donc ainsi résumer les résultats :

- En I960 on a constaté, en comparant deux groupes d'herbes, l'un normal, l'autre tétanigène, que ce qui les différenciait le mieux était :

a) Une teneur en azote non élaboré plus élevée dans les herbes tétanigènes ;

b) Une teneur en cellulose brute plus élevée dans les herbes normales;

c) Une teneur en magnésium plus élevée dans les herbes normales.

- En I96I on a constaté, en établissant la relation composition de l'herbeteneur en magnésium du plasma sanguin, que l'azote soluble de l'herbe a présenté une corrélation négative hautement significative avec le magnésium sérique des animaux.

\section{DISCUSSION}

I) Rôle des éléments minéraux autres que le magnésium

Il n'a été constaté aucune influence des éléments minéraux autres que le magnésium dans les deux séries d'observations (ont été dosés : $\mathrm{Ca}, \mathrm{P}, \mathrm{Mg}, \mathrm{Na}, \mathrm{K}$ ).

Le rapport $\mathrm{K} / \mathrm{Ca}+\mathrm{Mg}$ n'a pas présenté de relation avec le pouvoir tétanigène de l'herbe. On a d'ailleurs rappelé au début de cet article qu'il s'agit là d'une constatation très générale, et que même les auteurs qui émirent les premiers cette idée ne la soutiennent plus (KEMP, I960). La figure I permet de comparer l'histogramme de $\mathrm{K} / \mathrm{Ca}+\mathrm{Mg}$ d'herbes normales, douteuses et tétanigènes (résultats de I960) et de vérifier qu'ils se superposent étroitement tandis que la figure 2 permet de constater qu'il n'y a pas eu de corrélation entre le magnésium plasmatique et ce même rapport $\mathrm{K} / \mathrm{Ca}+\mathrm{Mg}$ (résultats de Ig6I).

On ne retrouve pas non plus l'influence du potassium considéré isolement, ou en rapport avec les protéines et le magnésium, comme dans les travaux les plus récents de KEMP (I960), mais conformément aux résultats de WERNER (I959), RoOK et WOOD (I960), BAKKER (I960) et RoOK et BALCH (I962). 
Dans la première série d'observations, le magnésium est apparu comme un facteur important, tandis que dans la deuxième série, le magnésium n'a pas eu d'action. Ce fait peut être en rapport avec l'origine géographique des prélèvements. Cette origine a été très variée (plusieurs clépartements du Nord et de la région parisienne) dans la première série, et beaucoup plus homogène (petite région à cheval sur la Flandre intérieure et la Flandre maritime) (lans la deuxième série. Il en résulte une plus grande dispersion des valeurs du magnésium de l'herbe dans la I $^{\text {re }}$ série (coefficient de variation de 34 p. 100 dans la première série contre 15 p. Ioo dans la seconde) plus propice à la mise en évidence de ce facteur.

On retrouve dans la littérature la même contradiction entre les auteurs qui

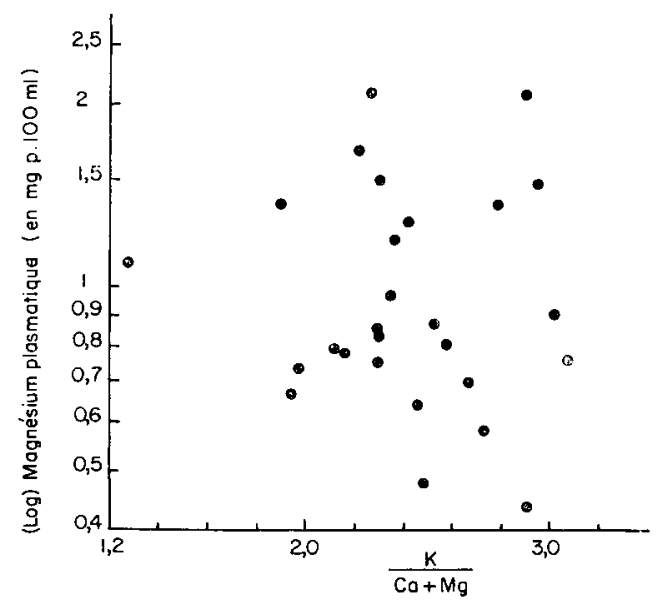

IIG. 2. - Relation entre le magnésium plasmatique et le rapporl $\mathrm{K}$ (a $+\mathrm{Hg}$ de l'herbe

\section{$\left.2^{\circ}\right)$ Rôle du magnésium}

ont travaillé sur une échelle géographique large (KEMP et 't HART, I957) et ceux qui ont opéré sur une échelle géographique restreinte (Rook et WoOD, Ig60, BAKKER, r960, WERNER, I959 et INGLIS, I960). Les premiers observent une action significative du magnésium qui n'apparaît pas aux seconds probablement parce que le magnésium a localement une marge de variation limitée.

\section{$\left.3^{\circ}\right)$ Rôle des matières azotées}

Dans les deux séries de résultats, les matières azotées de l'herbe ont été les facteurs les plus étroitement liés au déclenchement de la Tétanie d'herbage ou au niveau du magnésium plasmatique des bovins. Certains auteurs hollandais (REINDERS, I959, de VRIES, I959) ayant constaté que sur une prairie riche en trèfle les vaches avaient un magnésium sérique plus élevé que sur une prairie pauvre en trèfle, ont conclu (Rfinders, I959) que ceci pouvait être en relation avec le degré d'élaboration plus élevé de l'azote du trèfle par rapport à celıi des graminées jeunes ; nos résultats s'accordent asse $z$ bien avec cette conception quoique la teneur en magnésium élevée du trèfle puisse suffire à expliquer les résultats de ces auteurs. 


\section{$4^{\circ}$ ) Rôle de la matière sèche et de la cellulose brute}

La matière sèche de l'herbe n'a présenté aucune relation avec la 'létanie d'herbage ou l'hypomagnésiémie. La teneur en cellulose brute a eu une influence significative seulement dans la première série de prélèvements.

L'examen de l'ensemble des données permet de constater une différence importante entre les résultats des-analyses de I960 et ceux de I96r ; en I960 la teneur en cellulose brute de 1 'herbe tétanigène a été comprise entre $x_{3}$, I et 25,0 p. Ioo (moyenne I9, I p. IOO) tandis qu'en I96I la teneur en cellulose brute a été comprise entre 18,8 et 27,2 p. IOo (moyenne 22,8 p. IOO).

Cette constatation permet de suggérer l'utilité d'une quantité minimum de cellulose pour une assimilation correcte du magnésium.

Dans les conditions de nos observations, l'importance du facteur cellulose est très difficile à dégager et l'éclaircissement de ce point nécessitera de nouvelles recherches.

\section{CONCLUSIONS}

Parmi les substances dosées dans deux séries d'herbes tétanigènes, la teneur en azote, et particulièrement en a\%ote peu élaboré, s'est révélée être le meilleur témoin du pouvoir tétanigène de l'herbe. Dans la première série de dosages, les herbes tétanigènes se sont distinguées par une plus faible teneur en magnésium; cette relation n'a pas été observée dans la deuxième série, probablement par suite d'une localisation géographique plus restreinte les prélèvements. Mais il a été trouvé une relation négative entre le magnésium sérique et l'azote soluble de l'herbe. Une teneur faible en cellulose brute pourrait jouer un rôle favorisant de la Tétanie, tout au moins en dessous d'un certain seuil ; de nouvelles recherches à ce sujet seront nécessaires.

On n'a observé aucune relation entre le pouvoir tétanigène des herbes et leur

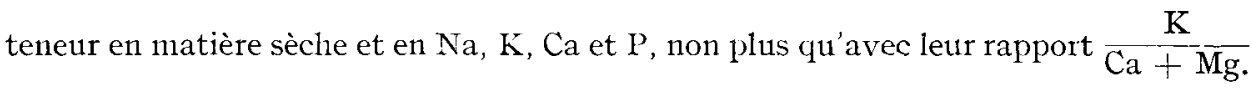
Reģu pour publication en janvier $\mathbf{1 9 6 3 .}$

\section{REMERCIEMEN'S}

Nous tenons à remercier vivement:

- M. Fauconnenu, du Jaboratoire des Métabolismes, C. N. R. Z., qui a bien voulu doser pour nous l'azote soluble des échantillons d'herbe.

- Messieurs les Docteurs Vétérinaires P. AGnet, de Monville, Seine-Mme ; P. Auquifr, de La Bassée, Nord; J. Avissle, de St-Pol-sur-Ternoise, Pas-de-Calais; P. Barisklle, de Bailleul, Nord; R. Basquin, de Roisel, Somme ; R. Baunun, de Ic Catelet, Aisne ; J. Bocquet, de Jaigle, Orne; M. Bossut, de Watten, Nord; M. Cinatelain, de Camieres, Nord ; M. Conyn et B. Hauven; 
de Hazebrouck, Nord ; A. Connette, de Audruicq, Pas-de-Calais ; M. Cornette, de Bergues, Nord, P. Courbet, de Pont-sur-Sambre, Nord; G. Courouble, d'Athies, Somme ; R. Davrainville, de Fresnoy-Folny, Seine-Mme; V. Delarue, de Cassel, Nord ; M. Depelsenaire, Le Quesnoy, Nord ; A. Dufour, de Vermand, Aisne ; C. IIoden, d'Abbeville, Somme ; R. Huguter, de Fourmies, Nord ; C. Lambert et Y. Besson, de Breteuil, Eure ; P. Lequin, de Solre-le-Château, Nord ; H. Lerede, d'Envermeu, Seine-Mme ; G. Menet, de Bermeries, Nord; J. Merlu, de St-Sulpice-de-Ham, Somme ; R. Pertriaux, de Cysoing, Nord ; R. Pierre, de Bosc-le-Hard, Seine-Mme ; J. Plateaux, de Fécamp, Seine-Mme ; C. Pronier, de Combles, Somme ; J. Richard, de Beauquesne, Somme; F. SAUVAGE, de Lumbres, Pas-de-Calais; L. SAX, de Bourg-Achard, Eure; G. Schmitr, de Houdan, Seine-et-Oise ; F. Seiliez, d'Arras, Pas-de-Calais ; C. WAtel, d'Inchy-en-Artois, Pas-de-Calais; P. Watremez, de Fresnoy-le-Grand, Aisne, grâce à l'obligeance et à la compétence desquels cette étude a pu être effectuée.

- Monsieur B. Vissac, de la Station de Génétique de l'I. N. R. A. dont les conseils nous ont été très précieux pour l'interprétation statistique des résultats.

\section{SUMMARY}

\section{GRASS COMPOSITION AND GRASS TETANY}

The relations between grass composition and either grass tetany symptoms or plasma magnesium in grazing dairy cows have been followed during the springs of 1960 and I96I.

$\left.I^{\circ}\right)$ Using R. A. FISCHER's discriminant function, the composition of two groups of grasses have been compared in spring 1960 . The first group was composed of 35 samples originating from normal pastures, the second of 45 samples originating from tetany-prone pastures. Analyses have been performed for dry matter, calcium, phosphorus, magnesium, potassium, sodium, crude fiber and non elaborated nitrogen.

Tetany-prone grasses were significantly lower in magnesium and crude fiber and higher in non elaborated nitrogen.

$2^{\circ}$ ) Using multiple regression equation, the plasma magnesium of grazing cows have been related to grass composition of 29 pastures during spring 196I. Analyses have been performed for dry matter, calcium, phosphorus, magnesium, potassium, sodium, crude fiber, crude protein and soluble nitrogen.

Soluble nitrogen was significantly related to plasma magnesium $(r=-0.543 ; \mathrm{P}<0.0 \mathrm{I})$.

Results of these two series of sampling have been compared and discussed.

\section{RÉFÉRENCES BIBLIOGRAPHIQUES}

Assoctation française de ZoOtechnie, 1959. Méthodes d'analyses préconisées. Réunion du 2 décembre. $27 \mathrm{pp}$.

BAKKER Y. T., I 960 . Teneur en magnésiun du sérum sanguin chez les vaches laitières dans différentes conditions (en hollandais). In $: D^{\mathrm{r}}$ Grashuis 25 jaar directeur $C$. $L$. O. controle.

Brouwer E., 195I. Étude sur les acides et les bases dans le foin, dans l'herbe normale et dans l'herbe provoquant l'hèmoglobinurie ou la tétanie d'herbage chez les bovins (en hollandais). Meded. Landbouwhogesch. Wageningen, 51, 91-1 12 .

BROUWER E., I952, On the base excess, the alkali alkalinity, the alkaline earth alkalinity and the mineral ratios in grass and hay with reference to grass tetany and other disorders in cattle. Brit. Vet. J., 108, I 23-r 3 I.

Brouwer E., VAN de Vliert A. J., I95I. Sur l'équilibre acido-basique dans le foin, dans l'herbe normale et dans l'herbe provoquant l'hémoglobinurie et la Tétanie d'herbage chez les bovins (en hollandais). Meded Landbouwhogesch. Wageningen, 51, 73-90.

BurT A. W. A., Thomas D. C., 196r. Dietary citrate and hypomagnesaemia in the ruminant. Nature, 192, Ir 93 .

Deltheil R., Hujon R., r959. Statistique mathémalique. 208 pp. A. Colin, Paris.

Annales de Zootechnie. - Ig63. 
Gueguen L., Rombauts P., ig6r. Dosage du sodium, du potassium, du calcium et du magnésium par spectrophotométrie de flamme dans les aliments, le lait et les excreta. Ann. Biol. anim. Bioch. Biophys., 1, 80-97.

HENDRIKS H. J., 1962. Quelques aspects biochimiques de la tétanie de nutrition (en hollandais). These Utrecht, I37 pp.

INGLis J. S. S., 1960. Studies on hypomagnesaemia in ruminants. 8th Internat. Grassland Congr., Reading, Section 58, p. I7-23.

KEMP A., I960. Hypomagnesaemia in milking cows : the response of serum magnesium to alterations in herbage composition resulting from potash and nitrogen dressings on pasture. Nether. J. Agric. Sci., 8, 28I-304.

KEMP A., 'T HaRT M. L., I957. Grass tetany in grazing cows. Nether. J. Agric. Sci., 5, I-18.

LaRvor P., I962. Relations entre la composition du plasma sanguin et les symptômes de tétanie chez les bovins. Ann. Zootech., 11, 135-149.

Naumans K., Barth K., i959. Chemische Untersuchungen im Weidetetaniegebiet des Niederrheins Landw. Forschung, 12, I 86-195.

ØDELIEN M., I960. La fumure peut-elle être la cause d'hypomagnésiémie et de tétanie chez les bovins? (en danois). Landbruksh. Instit. jordkultur. Bull., n 48 , I9 pp.

Peters A., 1960. Studien über den Zusammenhang zwischen Mängeln in der Winterstallfütterung hochleistender Milchkühe und der Grastetanie. Schriftenreihe der Landwirtschaftlischen Fakultät, Kiel, $\mathrm{n}^{\circ} 24,62 \mathrm{pp}$.

Reinderrs J. S., 1959. Hypomagnésiémie et alimentation (en hollandais). Tijdschr. Diergeneesk., 84, 630-642.

Rook J. A. F., BALCi C. C., I962. Magnesium metabolism in the dairy cow. III. The intake and excretion of calcium, phosphorus, sodium, potassium, water and dry matter in relation to the development of hypomagnesaemia. J. Agric. Sci., 59, 103-108.

Rook J. A. F., Wood M., i 960. Mineral composition of herbage in relation to the development of hypomagnesaemia in grazing cattle. J. Sci. Food. Agric., 11, 137-142.

SeEkLES L., I958. Tétanic de nutrition (Tétanie d'herbage) chez les bovins. Un problème somatique et écologique (en hollandais).

VERdeyen J., 1953. La relation entre la plante et l'animal. C.R. Recherches I. R. S. I. A., n ${ }^{0} 9,87-18 \mathrm{~s}$.

DE VRIES J. S., I959. Étude préliminaire sur l'importance des prairies riches en trèfle pour l'état sanitaire du bétail laitier (en hollandais). Kali (Amsterdam) (39), $33^{6-33^{8}}$ et $3^{68}$.

Werner W., I959. Über den Mineralstoffgehalt in jungen Weidefutter unter besonderer Berïcksichtigung des $\mathrm{K}:(\mathrm{Ca}+\mathrm{Mg})$ - Verhältnisses. Landw. Forschung, 12, I33-139. 